\title{
LA CONTRIBUTION DES “INTERVENTORES” ESPAGNOLS AU PROGRÈS DE L'ARCHÉOLOGIE NORD MAROCAINE (1912-1956)
}

\author{
LA CONTRIBUCIÓN DE LOS INTERVENTORES ESPAÑOLES EN EL PROGRESO \\ DE LA ARQUEOLOGÍA DEL NORTE DE MARRUECOS (1912-1956)
}

\author{
CHEDDAD A. MOHCIN*
}

\begin{abstract}
Résumé: Il serait injuste d'attribuer le progrès de l'archéologie nord-marocaine durant la période du protectorat (1912- 1935) aux seuls grands archéologues. Outre César Luis de Montalbán, le Père César Morán, Pelayo Quintero Atauri et Miguel Tarradell, on ne peut nier ou sous-estimer l'apport des "Contrôleurs" dans l'amélioration des connaissances relatives à ce domaine. A travers la lecture de quelques ouvrages et articles publiés durant cette période et la consultation des documents conservés au Musée Archéologique de Tétouan, les noms de certains de ces fonctionnaires s'imposent. Bien qu'ils soient peu connus, T. García Figueras, C. Pereda Roig, R. Touceda Fontenla et d'autres "Contrôleurs" méritent une reconnaissance à la hauteur des efforts qu'ils ont infatigablement déployés et les résultats qu'ils ont obtenus et qui restent encore à exploiter.
\end{abstract}

Mots-clés: Archéologie, contrôleurs, Protectorat, Nord du Maroc, Musée Archéologique de Tétouan, T. García Figueras.

En lisant les divers ouvrages, articles et rapports des archéologues espagnols qui ont travaillé au nord du Maroc durant l'époque du "Protectorat" (1912- 1956), nous nous sommes rendus compte du rôle remarquable
Resumen: Sería injusto atribuir el progreso de la arqueología en el norte de Marruecos durante el periodo del Protectorado (1912-1935) solo a los grandes arqueólogos. Así, al lado de César Luis de Montalbán, del Padre César Morán, de Pelayo Quintero Atauri y de Miguel Tarradell no podemos negar o subestimar la aportación de los Interventores en la mejora del conocimiento de esta disciplina. A través de la lectura de algunas obras y artículos publicados durante esta época y la consulta de documentos conservados en el Museo Arqueológico de Tetuán, se recuperan los nombres de algunos de estos funcionarios. Aunque sean poco conocidos, merece un reconocimiento, T. García Figueras, C. Pereda Roig, R. Touceda Fontenla, entre otros Interventores, para confirmar una realidad a la que no se le ha dado la importancia que merece. Debemos también ser conscientes de la calidad de los datos que nos han transmitido y que todavía no han sido completamente aprovechados.

Palabras claves: Arqueología, Interventores, Protectorado, Norte de Marruecos. Museo Arqueológico de Tetuán, T. García Figueras.

de certains fonctionnaires dans l'amélioration des connaissances acquises dans ce domaine, nous désignons essentiellement les "Interventores". Notre conviction s'est avérée incontestable quand nous avons

* Université Abdelmalek Essâadi, Faculté des Lettres et des Sciences Humaines, Martil (Marruecos).Correo-e: mcheddad2005@yahoo.fr. 
consulté ensuite les dossiers qui constituent l'Archive des documents écrits conservés au Musée Archéologique de Tétouan. Outre l'appui et la sécurité que ces fonctionnaires offraient aux chercheurs lors des opérations de fouilles ou de prospections (ICAME 1954: 18; Cabrera 2004: 192; Morán 1949: 35- 56), ils leurs fournissaient aussi de précieuses indications d'ordre scientifique en signalant l'endroit où subsistent les vestiges archéologiques, voire parfois même en ramenant au Musée quelques pièces. D'une manière analogue aux études qui ont prouvé l'implication de ces agents dans d'autres domaines (Nieto 1974; Villanova 2008: 507519 ; 2010), nous espérons à travers cet article élucider leur contribution au progrès de l'archéologie nord marocaine en évoquant diverses circonstances.

La présence des administrateurs espagnols à côté de leurs homologues marocains ne garantissait pas seulement la paix dans la région, mais elle permettait aussi la collecte de tous types d'informations. Les "interventores", ou les contrôleurs territoriaux sont avant tout des informateurs placés en contact de la population locale et desquels l'administration coloniale tirait ses renseignements fiables et détaillés à propos du territoire correspondant à leur cercle d'influence. La nature et le nombre des fonctions qu'ils assumaient a fait d'eux l'élément fondamental du bon déroulement de la politique espagnole au Nord du Maroc. Suivant une hiérarchie bien définie, le contrôleur local était mis à disposition du contrôleur régional qui dépendait lui aussi des ordres du Délégué des Affaires Indigènes, qui était à son tour attaché directement au Haut-commissaire de l'Espagne dans cette zone. Outre les qualités militaires, les contrôleurs devaient s'initier à des cours de langue, de géographie, d'histoire et d'autres sciences et arts très utiles pour répondre aux aspirations de leurs supérieurs (Figueras 1930; Delegación de Asuntos Indígenas 1935; Villanova 2000: 401-404; 2002). Malgré l'insuffisance des moyens, les conditions climatiques parfois différentes à celles de leur propre pays et la conversion de leur statut administratif à chaque changement des gouvernements, plusieurs contrôleurs ont fait une brillante carrière grâce à leur habilité, leur courage et l'envie d'améliorer leur situation(Villanova 2005: 93-111; 2006: 161-201; 2012a: 161-201; 2012b; 2013: 69-100; Mateo Dieste: 139-180; 2007: 643-670; Mateo Dieste et Villanova 2013: 595- 624).

L'exemple le plus éloquent de la réussite de ces fonctionnaires est sans aucun doute celui de Tomás García Figueras (1892-1981). Ce natif de la localité septentrionale du détroit de Gibraltar Jerez de la Frontera a entamé sa carrière militaire durant la phase d'occupation
(1921-1927) du nord du Maroc comme contrôleur des tribus de Beni Arous et de Soumata, puis contrôleur régional à Larache avant d'être promu en 1929 au poste de chef du Bureau Mixte dans la ville "internationale" de Tanger. Ensuite, après une courte étape à sa ville natale (1931-1936), il rejoint l'administration franquiste comme Secrétaire Général de la Haute Commissariat dans la zone du "Protectorat", puis Délégué de l'Economie, Délégué de la l'Education et de la Culture et Délégué des Affaires Indigènes entre 1952 et 1956. Andalou dévoué au courant africaniste, T. García Figueras est l'auteur des dizaines d'ouvrages et d'articles sur la politique espagnole au Maroc, les relations hispano-marocaines, l'histoire, l'économie, la religion et d'autres thèmes qui s'intéressent au Maroc (Figueras 1929; 1939; 1947b; 1947c: 284-289; 1955; 1957; [1952-1956]).

Nous évoquons ici ses deux articles parus en 1947 et en 1954 qui abordent les problématiques essentielles de la colonisation romaine au Nord du Maroc: le système défensif, les voies de communication et la pénétration à l'intérieur du pays (Figueras 1947a: 34-36; 1954: 331-335). La découverte des ruines du camp militaire romain de Souiyar et d'une tour de surveillance à Khandaq Hamar (où des blocs taillés et une meule romaine furent repérés en 1925) témoigne de la résolution de Rome à surveiller les terres fertiles de la vallée d'oued el Kharroub. A propos de la deuxième problématique, outre la possibilité d'un trajet traversant le col stratégique de Siwana, l'auteur affirme qu'une autre voie romaine passant à l'intérieur des terres par le site d'Afernoun reliait les sites de Tamuda et Lixus. Il présume enfin que grâce aux travaux de M. Tarradell et de C. Pereda, il n'est plus admissible de limiter l'implantaion romaine aux zones côtières. T. García Figueras ne manquait pas d'établir des analogies entre la présence romaine et celle espagnole à cette région en remarquant par exemple que certains camps militaires espagnols occupaient les mêmes endroits que ceux des Romains et en rappelant que les principaux objectifs de ces deux puissances se résumaient à l'exploitation des richesses agricoles et à assurer la passivité des tribus. Dans le second article, cet éminent politicien souligne l'importance de la collaboration des contrôleurs dans l'œuvre des archéologues: "Primero, por la posibilidad de señalar motivos de estudios; después por cooperar a la obra ya emprendida siguiendo las directrices técnicas o aumentando el área o la precisión de los reconocimientos". Après avoir vanté les mérites des contrôleurs dans ce domaine (en citant G. Hernández, C. Pereda et R. Touceda), il souligne l'intérêt des cours d'archéologie 
que Tarradell devait leur donner. L'une des quinze illustrations de ce même article offre le texte de l'inscription latine trouvée à Souiyar: $M / F$. NICE / NXXCIM / ZOIX / H. SPAN / CC N.

Un autre célèbre contrôleur est le basque Emilio Blanco Izaga qui a été formé à l'Académie militaire de Tolède avant d'être nommé en 1927 dans la zone orientale du "Protectorat". Son seul article qu'on peut classer dans le cercle de l'archéologie est celui qui concerne les rites funéraires préislamiques, publié dans la revue militaire África. A travers ses dessins et ses notes, E. Blanco nous a transmis une représentation mémorable sur les coutumes, les modes de vie, l'architecture et d'autres aspects de valeur artistique et ethnographique de la société rifaine entre 1927 et 1945 (Izaga 1964: 19- 21; Hart 1958; Hart, Romero et Nieto 1995; Romero 2009; 2014).

A vrai dire, l'ampleur de la contribution des contrôleurs en archéologie nord marocaine s'est manifestée dès les premières activités menées par C. Luis de Montalbán. Outre ses prospections dans la vallée d'oued Martil et sur les rives d'oued Loukkous, le premier archéologue espagnol au nord du Maroc a intervenu aussi dans la zone du Rif. Après avoir reçu le rapport du contrôleur de la tribu de Beni Ulichec (province de Nador), envoyé le 19 avril 1929 et l'apparition dans la presse locale d'un communiqué de presse (le 24 et 25 du même mois), la "Junta Superior de Monumentos" a sollicité à Montalbán d'y aller sur place pour élaborer une étude. L'archéologue a identifié un four avec des arcs en briques comme un monument servant à fondre des métaux et qui date de l'époque gothique (Montalbán 1930). Du même archéologue, nous signalons le rapport de ses prospections effectuées en compagnie du contrôleur de la tribu de Sumata (province de Larache), Carlos Jack Caruncho. Montalbán offre des informations sur les pratiques religieuses, la vie familiale et les coutumes de la population. Il signale aussi des galets cyclopéens, une grotte et des silex qui lui ont permis de parler des époques paléolithique et néolithique (Montalbán 1938). C'est à ce rapport que Quintero Atauri fait allusion en évoquant plusieurs sites préhistoriques et des constructions mégalithiques dans le territoire de Beni Guerfet et celui de Beni Aros et la remise d'un tronc d'une hache néolithique trouvé sur les terrasses de Sidi Mesuar au Musée de Tétouan (Quintero Atauri 1941: 38).

D'autres documents de l'Archive du Musée de Tétouan montrent l'ingérence des contrôleurs dans la planification des activités archéologiques, évidemment en accomplissant les décisions prises par leurs supérieurs.
Nous exposons à cet égard l'exemple du contrôleur de la région occidentale (Larache), M. Gonzalo Gregori qui fut nommé à ce poste en 1936.

Dans une première lettre adressée à C. Luis de Montalbán, il l'informe d'abord que par ordre du Secrétariat Général, les activités de la campagne de 1940 seront limitées à l'étude et à la conservation des ruines des fortifications portugaises de la Isla Graciosa. En même temps, il lui demande des précisions sur l'état actuel des recherches et des plans détaillés pour les travaux aux sites de Mzora, Ad Mercuri, Tabernes et Lixus.

Après avoir reçu la réponse et les propositions de l'Archéologue, G. Gregori envoie tout le dossier et une lettre datée du 29 avril 1940 à T. García Figueras.

Ce dernier examine minutieusement les documents et cite les remarques que G. Gregori devait faire à Montalbán (lettre envoyée le 1 mai 1940), parmi lesquelles:

1) la réponse de Montalbán, directeur des fouilles de la région occidentale, est insuffisante;

2) il doit inclure au programme une exploration que J. Martínez Santa Olalla, Chef du Service National de l'Archéologie, dirigera à Mzora;

3) le budget des travaux à la Isla Graciosa est limité à 3000 pesetas;

4) faire une demande à la Délégation des Affaires Indigènes pour autoriser à dix prisonniers d'Oued Laou de participer à ces travaux (comme à Gazzaza);

5) exprimer à Montalbán le dégoût que font ressentir ses constantes allusions au poste qu'il occupait avant l'instauration du régime franquiste et qu'il doit se soumettre aux instructions de l'Inspecteur des Fouilles de la zone.

Dans la même perception, nous trouvons à l'Archive du Musée une autre lettre du contrôleur territorial du Lucus (2 novembre 1943) se plaignant au Délégué de l'Education et de la Culture de la décision prise par Montalbán qui interdisait la "reparación en un abrevadero y paso de ganado" au site de Lixus. La réponse de l'Inspecteur des fouilles (Tétouan, 4 novembre 1943) reflète un désaccord inconcevable entre les deux archéologues puisque ce dernier autorise la continuation des travaux et “... llamar la atención del Director de Excavaciones de la región occidental por usurpación de atribuciones al disponer la paralización de las obras emprendidas, sin previa consulta al que suscribe...".

Nous signalons aussi quelques témoignages de Pelayo Quintero Atauri. Agé de 72 ans lors de sa désignation à Tétouan aux postes d'inspecteur des fouilles archéologiques au Nord du Maroc et conservateur du Musée (1939-1946), ses activités ont été presque 
réduites au site de Tamuda. Bien que ses excursions fussent rares, il faisait usage de plusieurs rapports qui arrivaient régulièrement au Service de l'Archéologie. Il mentionne, d'après les informations du contrôleur de Bab Taza, des restes d'une muraille (de $50 \mathrm{~m}$ de longueur sur un 1,50 $\mathrm{m}$ d'hauteur) qui remonte probablement à l'époque néolithique au village d'Amtras (tribu de Beni Seyyel, province de Chauen). A ses alentours, il mentionne des grottes encore inexplorées et les ruines d'anciennes localités connues par les habitants (Quintero Atauri 1941: 58).

De sa part, les écrits du Père César Morán Bardón nous donnent les noms de plusieurs contrôleurs. Pour son premier séjour dans la région de Beni Guerfet (août 1941), il mentionne Carlos Pereda qui avait envoyé une collection de matériel préhistorique au Musée de Tétouan (Morán 1941). Plus tard, lors de ses excursions dans la zone orientale, il fut tout le temps assisté par des contrôleurs (Morán 1949). Dès son arrivée à Villa Sanjurjo (l'actuelle Al-Hoceima), il est reçu par le contrôleur régional qui lui procure des lettres à présenter à ses secondaires. Le contrôleur local de la tribu de Beni Urriaguel, Antonio Servera Barceló le conduit à l'antique ville du Nekour où le lieutenant colonel Sánchez Pérez avait réalisé auparavant quelques travaux. Puis, en compagnie du contrôleur et d'un interprète, il visite la tribu de Beni Hadifa où il localise trois sites antiques: Casa Aiad, Tazrut Tucut et Tagsab. De Boudinar, il s'est rendu avec le contrôleur, au village de Trifa où ils furent accueillis chez un notable autochtone. Le contrôleur de Zaio lui indique des ruines sur la colline appelée Kasba située entre les embouchures d'oued el Hach et oued Marsud.

Dans la région occidentale, C. Morán et Gustavino Gallent réalisent plusieurs prospections à la recherche des traces des voies romaines (Morán et Gustavino Gallent 1948). Ils visitent le site d'Ad Mercuri (près de Had el-Gharbia) en présence du contrôleur de Miquel, puis ils partent, accompagnés du contrôleur, à la visite de Tabernae. Ils se rendent ensuite à Lixus avec le colonel Galera et le lieutenant-colonel Sánchez Pérez. En apprenant les renseignements du contrôleur d'Azila, Álvarez Amado, à propos des ruines situées à environ $8 \mathrm{~km}$ au Sud Est de cette ville, les auteurs (guidés par le commandant San Pedro) inspectent le site appelé El Jareb (les ruines), entre les villages d'El Homar et d'Oulad El Arbi, où ils découvrent sur une petite colline des vestiges antiques (céramiques, briques, ruines de muraille et d'habitats,...). Doutant de l'identification de Qsar el-Kebir à l'antique colonie
d'Oppidum Novum, les auteurs se rendent, guidés par le lieutenant-colonel Antón Orejuela, au mont Gani puis à la petite colline appelée Cunna où abondent des vestiges romains (céramique, amphores, briques...). Accompagnés par un Caïd du Groupe des Réguliers, ils visitent aussi d'autres ruines à Snadla, à environ $5 \mathrm{~km}$ à l'Ouest de Qsar el-Kebir et à quelques kilomètres du pont de Kerma “... El mismo informador nos dice haber visto, allá por el año 1912, vestigios de puente sobre el Lucus, que pasa al N. de la población". Lors d'une rapide excursion sur la deuxième voie reliant Tamuda et Ad Abilem, les auteurs, accompagnés du contrôleur de Malaliène, s'arrêtent à Cabo Negro (Ad Aquilam Maioremde l'Itinéraire d'Antonin), à Punta de Castillejos (Ad Aquilam Minorem) où des restes d'un ancien bâtiment carré avec des arcs semi-circulaires et des murs de mortier en pierres et en chaux existent tout près de la mosquée. En se dirigeant de la ville de Chauen à oued Laou (en compagnie des contrôleurs de Chauen et de Talambot), les auteurs signalent trois ponts utilisés au Moyen Age à proximité de ce dernier lieu et qui pourraient avoir succédé à d'autres plus anciens. Ils signalent enfin que le contrôleur de Ben Qarrich avait remis à la Délégation de l'Education et de la Culture un fragment d'une statue antique représentant une tête féminine trouvé quand on construisait le dispensaire de cette localité en 1946.

Si dans ces cas les contrôleurs n'ont eu qu'un rôle secondaire, dans d'autres c'est eux qui ont eu l'honneur de découvrir d'importants sites ou pièces archéologiques. C'est ainsi que les seules gravures rupestres connues dans la région, celles de la grotte nommée Magara, au village de Kasba furent localisées grâce au contrôleur de la tribu de Beni Issef et de Beni S'kar, E. García Hernández (García Hernández 1941: $300-$ 302; Martínez Santa Olalla 1941: 438-442; 1942: 2224; Quintero Atauri 1942: 76). Elles sont situées à trois heures de marche de Meserah, le siège le plus près de l'administration espagnole. Les dimensions de la grotte sont modestes (environ 1, $60 \mathrm{~m}$ de largeur, $1,50 \mathrm{~m}$ de hauteur avec un plafond de $1,80 \mathrm{~m}$ sur $2 \mathrm{~m}$ de profondeur) et les peintures ne sont pas en très bon état de conservation: des lignes en points rouges, un homme sur un cheval, suivi de trois autres personnes marchant à pieds. Ce contrôleur a publié un autre article où il expose ( 6 photos à l'appui) les résultats de ses explorations à plusieurs grottes au village de Kasba, à piedra Talaka el Arab, à Magara d'Aïn el Hayar, à Khalwa de $\mathrm{M}^{\mathrm{y}}$. Abdelkader, etc. (García Hernández 1942: 14-15). 
Toujours dans la zone occidentale du "Protectorat", le contrôleur de la tribu de Beni Messawar, San Pedro, signale au mois d'avril 1941 la découverte à Dar Chawi des vestiges d'édifices, des céramiques et des monnaies romaines (une grande pièce en bronze de Trajan et deux autres de Dioclétien). Quintero Atauri identifie ce site à la colonie romaine de Iulia Campestris Babba (Quintero Atauri 1941: 39). Les circonstances de la découverte sont révélées par une lettre du contrôleur régional de Jebala (¿San Pedro?) datée du 28 avril 1941 qui parle de la construction "de una fábrica de crin vegetal propriedad de D. Rafael Benet, al lado de la carretera general".

Grâce au rapport du contrôleur de la région occidentale concernant des ruines romaines (muraille du camp, nécropole, restes d'édifices, un four, tegulae, briques...) au village d'El Homar (à $8 \mathrm{~km}$ au nord d'Azila), Quintero Atauri suppose qu'elles correspondent à la station routière Ad Novas distante d'Oppidum Novum par trente-deux milles. Il estime qu'il serait profitable de mener des fouilles aux sites signalés par le contrôleur régional de Larache et par le Commandant San Pedro (Quintero Atauri 1942: 13). Certainement, Quintero Atauri utilisait le télégramme du contrôleur d'Azila envoyé à T. García Figueras le 5 juillet 1941 (conservé aux Archives du Musée Archéologique de Tétouan):

"En el día de ayer regreso por segunda vez de Tetuán Interventor Adjunto sin cobrar cerca de cinco mil pesetas que se deben por excavaciones Don Pelayo puso toda clase de dificultades y necesito que este descubierto de la Intervención sea compensado no haciéndose más pagos hasta tanto resuelva V. E.

Día de hoy fue hallada ciudad Ad Nova entre poblado de Homar y Uad el Arbi cercanías del Tenin, hallándose amplio castro necrópolis con sepulturas a la vista y resto de edificaciones. Saludole respetuosamente".

Au sujet de la nécropole de Raqqada, située au voisinage de Lixus, nous lisons un communiqué du contrôleur régional de Larache, paru dans le journal Marruecos (3 juillet 1942) qui parle de la découverte de quelques objets, probablement puniques à l'embouchure du Lukous, au lieu dit Campamento del General Silvestre (tribu de Racada). Il s'agit de tombes construites de blocs taillés, peut-être extraits de la carrière Punta Negra, sans mortier et avec une magnifique dalle à la couverture. A l'intérieur n'ont apparu ni restes humaines ni céramiques “... solo un par de piezas en forma de torcues, de los cuales únicamente se ha conservado una, pues la otra fue rota... El metal empleado, parece ser el llamado 'electrum' de la época púnica...”. Il espère que des futures fouilles apportent plus d'éclaircissements.

Nous devons également dans ce contexte rappeler la découverte par le contrôleur territorial de Yebala, Luis Carvajal Arrieta (en 1948) à Qsar es-Seghir d'une inscription et des restes de l'époque portugaise ainsi que la découverte par le contrôleur de la tribu d'Anjra, Epifanio González García (en 1952), aux environs de Souk el Khemis de deux inscriptions libyques qui furent envoyées au Musée Archéologique de Tétouan.

Un autre contrôleur qui mérite beaucoup de respect pour ses efforts considérables dans le progrès des connaissances archéologiques dans la zone nord marocaine est R. Touceda Fontenla. Outre ses multiples écrits sur les croyances et les coutumes de la population locale, l'histoire et la géographie de la région ou sur d'autres thèmes (Touceda Fontenla 1951: 92; 1952; 1955; 1957a: 421-423; 1958: 74-79; 1959b: 222224; 1960: 426-430; 1961: 214-218; 244-248; 286290; 1962: 11-13; 9-11; 1967), ce contrôleur d'Arbâa d'Ayyacha (mort le 16 juin 1998) a laissé quelques intéressants rapports archéologiques. T. García Figueras lui avait demandé pour sa communication au I Congrès Archéologique du Maroc espagnol, de visiter le camp de Souiyar et le site de Khandaq Hamar afin de prendre des notes et des photos. Voici les textes de la lettre manuscrite envoyée à ce sujet par le contrôleur à $\mathrm{M}$. Tarradell, le 12 juin 1953 et la réponse de ce dernier:

\footnotetext{
"Muy señor mío. Le envío las piedras que descubrí en unas ruinas inmediatas a esta oficina que juzgo de interés para ese museo. En el sitio donde han sido halladas existen los cimientos de una "fortaleza" o algo parecido y aunque los naturales dicen que es de los "portugueses" pudiera ser muy bien que se tratara de algo fenicio o romano.

Existen innumerables piedras talladas, he medido una de 170 de largo y alguna en forma de capitel que tengo fotografiado, etc.

Si interesa algún otro dato para ese museo, puedo darlo gustoso.

Las piedras han sido halladas en un sitio denominado "Suiar" de esta fundición de Arbaà Aixa, a orillas del rio Jarrub, a la altura del km. 60 de la carretera Tetuán- Larache.

Con más detalles informo igualmente a D. Tomas García Figueras, que me hizo el encargo sobre otras ruinas -al parecer de origen romano- que se hallan a $3 \mathrm{~km}$ de estas a que me refiero.

Sin otra particular le salude atentamente su afmo s.s"
}

Réponse de M. Tarradell: 
“Tetuán, 12 de junio de 1953

Sr. D. Ramón Touceda Interventor del Arbaa de Laiacha

Muy Sr. mío;

Enviadas por Vd. se han recibido tres piedras, una de ellas con inscripción latina, de que ha sido portador el mejasni Mohamed B. Mohamed B. Ali, quien me ha entregado también su carta. Por los detalles que en ella me da parece tratarse de unas ruinas importantes, a juzgar por la extensión. Dentro de unos días espero ir a Larache y aprovecharía para saludar a Vd. y, si es posible dar un vistazo a estas ruinas.

El mejasni quería permanecer aquí hasta obtener las fotos cuyos negativos traía, pero yo le he aconsejado dejarlo aquí y mañana se lo mandaremos nosotros todo con alguna Valenciana. Incluso lo hemos llamado por teléfono y nos han dicho estaba Vd. el Had de la Garbia.

Le quedo muy agradecido por todo. Gracias a la labor de Vds., beneméritos Interventores, podemos ir salvando multitud de reliquias del pasado que sin su valioso concurso se perderían.

Como le digo, espero saludarle muy en breve y entre tanto sabe puede disponer de su $\mathrm{afm}^{\circ}$. s. s.”.

Nous sommes convaincus que R. Touceda a bénéficié largement des enseignements de Tarradell auquel il fait parfois référence pour certifier la datation de certains objets ou l'identification des monuments. Son article publié dans la revue franciscaine Mauritania prouve un niveau convenable et constitue une source d'informations rarement utilisée par les archéologues (Touceda Fontenla 1959a: 99-107).

A travers des prospections menées dans un territoire peu fréquenté, il reconnait plusieurs sites antiques:

- Au village de Zeituna (tribu de Beni Ider), sur la rive droite d'oued el Kharroub, il mentionne des pierres taillées de grandes dimensions et plusieurs fragments de la céramique romaine.

- A 1 km à l'est, à proximité de Le Tenin, il cite une nécropole romaine où il compte trente tombes et fouille une seule. Non loin, il signale des fragments de la céramique romaine dont un de la terra sigillata.

- A moins d'un kilomètre, sur la rive du fleuve, il repère des blocs taillés et plusieurs fragments de la céramique romaine.

- Il visite aussi les ruines de Khandaq Hamar signalées par García Figueras en 1926 et signale une meule romaine au lieu-dit Talaya occupé par un poste militaire espagnol. Il effectue deux sondages de deux mètres de profondeur qui ont révélé l'existence de troncs de mur formant l'angle d'un édifice (il parle de trois ou quatre édifices).
- Il se rend également au camp de Souiyar où il signale la découverte d'une inscription latine: $M / F$. NICE/ NXXIM/ZOIX/ H. SPAN/CCN, qui fut expédiée au Musée de Tétouan, ainsi que des céramiques, des morceaux de chapiteaux et d'autres vestiges de l'époque romaine.

- Non loin de Tlata de Jebel Hebib, il localise un autre poste militaire romain situé sur une petite élévation (à environ $5 \mathrm{~km}$ de Souiyar). La présence des céramiques et des blocs de mortier romains au site appelé Rokba el Gozal, presque en face de la nécropole de Le Tenin, à l'endroit occupé par un poste militaire espagnol a incité l'auteur de conclure que: "La modalidad de la guerra que Roma llevó a cabo en Marruecos, la de penetración, fue la misma que utilizó España en su misión de pacificación...”.

- Il signale aussi des céramiques et des murs à Aïn Mazzos (à $2 \mathrm{~km}$ du village de Quesiva), surtout sur une petite colline appelée par les habitants "Colina de las tejas" où des fragments de la céramique romaine couvrent le surface du sol. Après que des habitants lui informent de l'existence d'un ancien temple, il réalise des fouilles qui lui ont permis de repérer un édifice romain de forme rectangulaire.

- Un peu plus loin, auprès du village d'Oulad Boujemâa, l'auteur note des murs bâtis par des grands blocs taillés qui semblent appartenir à trois édifices et recueille des fragments de la céramique romaine: il croit avoir localisé un poste militaire romain dominant la vallée d'oued Ayyacha.

- A $1 \mathrm{~km}$ de ce site, sur le bord du fleuve, il signale de nombreux fragments de céramiques, des tuiles, des briques romains mais sans apercevoir les vestiges d'aucune structure. Si l'existence à cet endroit d'un atelier de poterie reste hypothétique, l'auteur est persuadé que c'est par là que passait la voie romaine conduisant à l'intérieur du pays et dont un tronc fut reconnu entre Tnine de Sidi Yamani et la localité de Dar Saidi.

- A l'emplacement consacré au marché hebdomadaire d'Arbâa d'Ayyacha (précisément à l'endroit réservé à la vente du charbon), un sondage a permis à $\mathrm{R}$. Touceda de récolter des fragments de la céramique romaine et de distinguer des murs perpendiculaires dont un tronçon mesure 8 mètres. Il évoque aussi à l'extrémité du même Souk, une plateforme d'environ $3 \mathrm{~m}^{2}$ avec des bords d'environ $20 \mathrm{~cm}$ de hauteur que Tarradell identifie comme un bain romain.

- Plus loin de cette zone, l'auteur mentionne des ruines romaines à Koudiat Chuna et à Koudiat Tex (près du souk el Khemis de Sidi Ali, à droite de la 
piste allant vers Beni Ider). Sur la première, où existait un poste militaire espagnol entre 1923 et 1926 , furent signalés des grands blocs taillés et de la céramique qui indiquent peut-être l'existence d'une garnison romaine. Ces deux positions, établies à environ $70 \mathrm{~km}$ de la côte par le trajet le plus court, confirment l'implantation romaine dans la zone montagneuse du Nord du Maroc.

En conclusion, l'auteur estime que tous ces sites doivent être etudiés de manière plus sérieuse.

Dans un autre article, R. Touceda signale dans la région de Had el-Gharbia des rochers allongés sur une dizaine de mètres représentant ainsi des murs cyclopéens de $16 \mathrm{~m}$ de longueur sur $9 \mathrm{~m}$ de largeur en forme d'un rectangle orienté du nord au sud. Les habitants les nomment Hammam. Devant l'incertitude de les identifier à un lieu de sacrifice humain, l'auteur considère qu'ils peuvent au moins servir pour la localisation de la station Ad Novas "que Tissot suponía a las alrededores del Tnin de Sidi Yamani...?” (Touceda Fontenla 1962: 241-245).

Outre ses articles parus dans la revue Mauritania, R. Touceda Fontenla a participé aussi au I Congrès Archéologique du Maroc espagnol organisé en 1953 avec deux communications (Touceda Fontenla 1954: 461$468 ; 511-514)$. A ce Congrès qui a réuni les plus grands spécialistes du domaine à l'époque, un autre contrôleur était présent: Carlos Pereda Roig (1909-1978). Dès 1927, il rejoint la zone du "Protectorat" comme interprète. Entre 1934 et 1956, il occupe le poste de contrôleur. Après l'indépendance du Maroc, il demeura à Tanger jusqu'à sa mort comme formateur des fonctionnaires de la nouvelle administration. Parmi ses célèbres travaux, nous rappelons un recueil de 651 dictons poétiques recueillis dans diverses zones de la région de Jebala et qui ne fut publié que récemment (Pereda Roig 1939; 1941; 2014).

Sa communication au célèbre congrès archéologique concerne un trajet peu exploré qui s'étend de l'embouchure d'oued Laou à la plage de Jebha, identifiée par l'auteur au comptoir appelé par les sources classiques Taenia Longa (Pereda 1954: 443- 460). En se déplaçant d'une plage à l'autre, parfois en voie maritime, C. Pereda qui fait souvent référence au rapport de Montalbán intitulé Viaje de estudio desde el rio Lau al rio Nekor, localise plusieurs sites qui remontent aux époques préislamiques: "...restos de una alberca romana al pie del montículo Sur de Esteha; restos de las balsas romanas descubiertas en Yenanich; calzada romana de Tagsa;...". Au terme de son exposé, l'archéologue français R. Thouvenot souligne l'énorme intérêt des informations alors qu'Almagro déclare: "La conferencia del Sr. Pereda honra a los interventores...". La copie de ce rapport qui est déposée à l'Archive du Musée Archéologique de Tétouan est très mutilée, il n'en reste que l'album contenant les 47 photos qui illustrent ces explorations.

Un autre article de C. Pereda s'attache à la localisation du site romain Oppidum Novum. Des informations orales ont incité l'auteur à se rendre au lieu nommé Dar el Majzen, au sud de Fondak Eyyauhar, où il remarque l'existence des briques, des amphores et des céramiques romaines. Il décrit les structures visibles en surface, réalise de simples fouilles avant de revisiter le site accompagné du P. César Morán. Ces nouvelles découvertes l'ont permis de localiser Oppidum Novum à quelques kilomètres loin de la ville actuelle de Qsar el-Kebir (Pereda Roig 1942: 70-71).

Dans la zone orientale du "Protectorat", nous mentionnons d'abord Andrés Sánchez Pérez. Contrôleur de Beni Ammart et Sanhaja dès 1926, puis à partir de 1933 de la puissante tribu de Beni Urriaguel avant qu'il soit nommé en 1942 au poste de contrôleur régional du Rif. A cette même année, il fait connaissance à l'anthropologue américain, Carleton Stevens Coon qui avait publié un travail sur l'ethnographie des tribus du Rif. En 1946, A. Sánchez occupe le poste de contrôleur régional à Larache (région du Lucus). Ce militaire qui fut aussi professeur de la langue arabe, a écrit plusieurs ouvrages sur les tribus du Rif et ses célèbres personnages comme Abdel-Karim el Khattabi (Sánchez Pérez 1926; 1950-1951: 95-104; 1964: 15). A propos de ses activités archéologiques, on signale qu'après que des travaux routiers sur la rive gauche du fleuve Nekor aient dégagé des vases entiers en 1929, il réalise des fouilles en 1934 et publie ultérieurement un rapport basé aussi sur les récits historiques d'Ibn Khaldoun, de Léon l'Africain, de Marmol,... relatifs à la ville homonyme et les deux autres importantes villes médiévales de cette région: Al-Mazamma et Badis (Sánchez Pérez 1951- 1952: 31-47). Nous savons aussi que lors de sa mission à Larache, il a envoyé à plusieurs reprises des lots monétaires provenant des sites de Lixus et d'Ad Mercuri au Musée de Tétouan (Sánchez Pérez 1948: 36). D’autres rapports du même auteur se trouvent dans la Collection de T. García Figueras à la Bibliothèque Nationale de Madrid: Las exploraciones arqueológicas de Beni Urriaguel (BN, Biblioteca García Figueras. Miscelánea, tomo LIII, 38: 169); El Cromlech de Mezora (BN, Biblioteca García Figueras. Miscelánea, tomo LXIII, 178: 217) et Arqueología en el Lukus. Lixus (BN, Biblioteca García Figueras. Miscelánea, tomo LXIII, 178: 246). 
Pour cette même zone, Quintero Atauri signale dans son troisième mémoire consacré au fouilles de Tamuda, que des petits objets en bronze (des fragments d'un mors de cheval, des fibules et des supports de poignard) provenant des ruines de Badis de l'époque wisigothe furent livrés par l'Intervention Territoriale au Musée archéologique de Tétouan. Il note aussi la remise au Musée de trois monnaies en or provenant des ruines de Nekor, étudiées par le numismate Mateu y Llopis (Quintero Atauri et Giménez 1944: 27).

Dans le même rapport, Quintero Atauri mentionne que l'Intervention Territoriale du Rif, assistée par le Père Antonio Gauna, de la Mission Franciscaine de Villa Sanjurjo, a recueilli à proximité de l'ancien camp d'Ikauen (tribu de Ketama) plusieurs objets lithiques dont quelques-uns sont d'un grand intérêt (11 haches polies de différentes tailles, des pierres polies, une flèche et un grattoir taillés en quartzite de la période néolithique et une hallebarde de pierre polie de $20 \mathrm{~cm}$ de longueur et 4 au largeur). Les haches, le grattoir et la flèche furent récoltées fortuitement à Beni Urriaguel et à Bocoia, ils témoignent peut-être de l'existence d'importants sites néolithiques, inconnus des archéologues (Quintero Atauri et Giménez 1944: 26). Ces indications concordent avec le document conservé à l'Archive du Musée de Tétouan: la lettre envoyée le 11 octobre 1952, par le contrôleur de Villa Sanjurjo (l'actuelle Al-Hoceima), Antonio Fornes Andrés en référence à l'envoi d'une caisse contenant des objets extraits des ruines de Bades.

Nous devons insister à ce propos que c'est surtout après la diffusion du questionnaire archéologique élaboré par J. Martínez Santa Olalla (Inspecteur Général des Fouilles Archéologiques) que l'intérêt des contrôleurs aux restes préislamiques s'est intensifié plus amplement (Barradas, Alonso del Real et Martínez Santa Olalla 1940). Des dizaines de fiches de réponses parvenues de diverses zones sont encore conservées au Musée, elles méritent une étude approfondie. Certaines d'entre elles mentionnent des grottes, des sépultures, d'anciennes ruines non encore explorées et qui sont illustrées par des photos, des croquis...

\section{CONCLUSION}

Il ressort alors que l'étude de la contribution de ces administrateurs, inconnus par la plupart des chercheurs actuels, est essentielle pour la compréhension de l'évolution des connaissances archéologiques au nord du Maroc durant la période du "Protectorat". Nous n'avons pas voulu insister ici sur la véridicité et l'exactitude des renseignements présentés par les contrôleurs puisque leurs écrits ne représentaient en effet qu'une matière brute que les archéologues devaient examiner. C'est vrai que le niveau des connaissances de la plupart d'entre eux n'était pas suffisant pour identifier, dater et élaborer une synthèse historique basée sur les trouvailles archéologiques, mais nous devons en parallèle reconnaitre leur courage à mener des prospections dans des territoires difficilement accessibles, leur détermination à réaliser des fouilles bien qu'ils soient superficielles et surtout leur rendre hommage pour la documentation, riche et en grande partie inédite, qui nécessite un examen attentif. Leurs intuitions formulées dans des rapports très abrégés se sont avérés parfois corrects: comme ils le prévoyaient certains sites signalés et qui n'ont été fouillés que récemment (Sidi Boulenoire -prés du village d'Oulad Boujemâa-, Raqqada,...) se sont révélés d'une grande importance pour l'histoire de la région. Ils ont eu également le mérite d'aborder des problématiques encore discutées comme la localisation des sites d'Ad Novas ou d'Oppidum Novum, le tracé des voies romaines, etc.

Nous ne devons plus douter de leur grandiose assistance aux historiens et aux archéologues qui dépendaient largement de leurs informations. Si les trois grandes figures, Montalbán, Quintero Atauri et Tarradell se situent au devant de la scène archéologique nord-marocaine à cette époque, alors que le Père C. Morán Bardón, M. Gómez Moreno et d'autres qui ont rédigé quelques articles viennent au second plan, il est impératif d'ajouter à cette liste les noms de plusieurs contrôleurs. Grâce à certains de ces délégués officiels qui géraient les affaires des tribus comme des gouverneurs et qui ont eu la passion des explorations et des découvertes que le rythme des activités archéologiques s'est accéléré, surtout sous le régime dictatorial de Franco. Dans son premier article sur l'archéologie marocaine, Tarradell souligne les difficultés politiques et financières que les chercheurs devaient affronter, la négligence des habitants aux monuments antiques et le rôle considérable des contrôleurs dans plusieurs découvertes (Tarradell 1949: 80).

\section{BIBLIOGRAFIE}

Blanco Izaga, E. (1946): "Noticias sobre arte funerario africano". Africa 52: 19-21.

Bravo Nieto, A. (1974): "La genèse d'un style colonial: l'architecture rifaine dans le Maroc espagnol". Revue du Monde Musulman et de la Méditerranée 73: 167-182. 
Cabrera, A. (2004): Magreb-el-Aksa. Recuerdo de cuatro viajes por Yebala y por el Rif, prólogo de $\mathrm{M}$. Hernando de Larramendi. Madrid, Editorial Ibersaf.

I Congreso Arqueológico del Marruecos Español (Tetuán, 1953) (1954), Tetuán, Imprenta Cremades.

Montgomery Hart, D.; Moga Romero, V. y Bravo Nieto, A. (eds.) (1995): Coronel en el Rif: una selección de su obra, publicada e inédita, sobre la estructura sociopolítica de los rifeños del Norte de Marruecos. Melilla, UNED.

Cuevas, T. de (1885): "Ruinas romanas del reino de Fez (Marruecos)". Boletín de la Real Academia de la Historia VII: 40- 45.

García Figueras, T. (1929): Acción de España en Marruecos. Madrid, Imprenta Municipal.

García Figueras, T.(1930): Datos y organización de las intervenciones. Marruecos (Protectorado Español), Inspección General de Intervención y Tropas Jalifianas.

García Figueras, T. (1939): Notas sobre el Islam en Marruecos. Larache, Artes Gráficos Bosca.

García Figueras, T. (1947a): “La ocupación romana del N. O. de Marruecos", dans Homenaje a Julio Martínez Santa-Olalla II. Actas y Memorias de la Sociedad Española de Antropología, Etnografía y Prehistoria, XXII, 1-4: 34-36.

García Figueras, T. (1947b): África en la acción española. Madrid, Ediciones de la Dirección General de Marruecos y Colonias y del Instituto de Estudios Africanos.

García Figueras, T. (1947c): "Las etapas de la pacificación”. África. Revista de Acción Española 68-70: 284-289.

García Figueras, T.(1954): "La incógnita del valle del Jarrub. Las ruinas romanas de Suiar", dans I Congreso Arqueológico del Marruecos Español (Tetuán, 1953): 331-335, Tetuán, Imprenta Cremades.

García Figueras, T.(1955): Marruecos: la acción de España en el Norte de África. Tetuán, Editora Marroquí ( $4^{\mathrm{a}}$ ed.).

García Figueras, T. (1952-1956): Cartas a los Interventores, document dactylographié, s/1, s/a (¿1950?).

García Figueras, T.(1957): España y su protectorado en Marruecos (1912-1956). Madrid, Instituto de Estudios Africanos, Consejo Superior de Investigaciones Científicas.

García Hernández, E. (1941): “Un abrigo con pinturas rupestres en Beni Issef”. Mauritania 14: 300-302.

García Hernández, E. (1942): "Prehistoria norte africana. Covachos y piedras en Kasba". Mauritania 170: 14-15.
Martínez Santa Olalla, J. (1941): "Las primeras pinturas rupestres del Marruecos español”. Actas y Memorias de la Sociedad Española de Antropología, Etnografía y Prehistoria 16, 3- 4: 438-442.

Martínez Santa Olalla, J. (1942): "Die höhle Yebel Kasba in Neues über prähistorische Felsmalereien in Frankreinch, Spanien und Marokko". Jahrbuch Prähistorische und Etnographische Kunst 15-16: 22-24.

Mateo Dieste, J. L. (2002): "La oficina de intervención como espacio de interacción socio-política entre el murāqib y la cabila: de la ideología colonial a las prácticas cotidianas", dans F. Rodríguez Mediano et H. de Felipe (eds.), El Protectorado español en Marruecos. Gestión colonial e identidades e identidades: 139-180. Madrid, Consejo Superior de Investigaciones Científicas.

Mateo Dieste, J. L.(2007): “El interventor y el caíd. La política colonial española frente a la justicia marroquí durante el protectorado de Marruecos (19121956)". Hispania LXVII, 226: 643-670.

Mateo Dieste, J. L. et Villanova, J. L. (2013): “Les interventores du protectorat espagnol au Maroc. Contextes de production d'une connaissance politique des cabilas". Cahiers d'Etudes Africaines 211, 3: 595-624.

Moga Romero, V. (2009): El Rif de Emilio Blanco Izaga: trayectoria militar, arquitectónica y etnográfica en el Protectorado de España en Marruecos. Melilla, Edicions Bellaterra, UNED-Melilla.

Moga Romero, V. (ed.) (2014):La Atlántida rifeña de Emilio Blanco Izaga. La impronta de un militar español en Marruecos, 1927-1945. Melilla, Servicio de Publicaciones Ciudad Autónoma de Melilla.

Montalbán, C. L. de (1930): Memoria sobre la ciudad de Cazaza de la Cabila de Beni-Bugafar y del horno de fundir metal encontrado en el poblado de Bohemana de la kabila de Beni-Ulichec. Larache, Alta Comisaría de España en Marruecos.

Montalbán, C. L. de (1938): Exploraciones arqueológicas en la Kabila de Sumata. Larache.

Morán, C. (1941): El Paleolitico de Beni Gorfet (Marruecos), Junta Superior de Monumentos Históricos y Artísticos, $n^{\circ} 4$. Larache, Instituto General Franco.

Morán, C.(1949): “Antiguas poblaciones del Rif”. Archivos del Instituto de Estudios Africanos: 35-56.

Morán, C. y Gustavino Gallent, G. (1948): Vías y poblaciones romanas en el Norte de Marruecos. Alta Comisaría de España en Marruecos, Delegación de Educación y Cultura, $\mathrm{n}^{\circ} 11$. Madrid, Otice.

Montgomery Hart, D. (1958): Emilio Blanco Izaga and the Berbers of the central. Tetuán, Imprenta Cremades (= Tamuda 6, 2 [1958]: 171-237). 
Pereda Roig, C. (1939): Los hórreos colectivos de Beni Sech-Yel.Ceuta, Imprenta Imperio.

Pereda Roig, C.(1941): Concordancia de los calendarios de la Hégira y de la Era Cristiana, desde el año 1 de aquella hasta el 2000 de esta. Tetuán, Instituto General Franco.

Pereda Roig, C.(1942): “¿Nuevas ruinas romanas en Alcazarquivir?”.Mauritania 172: 70-71.

Pereda Roig, C.(1954): "Itinerarios arqueológicos de Gomara La costa", dans I Congreso Arqueológico del Marruecos Español (Tetuán, 1953): 443-460. Tetuán, Imprenta Cremades.

Pereda Roig, C.(2014): Coplas de la región de Yebala (Norte de Marruecos), presentación, estudio, notas, glosario y bibliografía de F. Moscoso García. Barcelona, Edicions Bellaterra.

Pérez de Barradas, J.; Alonso del Real, C. y Martínez Santaolalla, J. (1940): Investigación cientifica de Marruecos. I Cuestionarios de Etnología, Lingüística y Arqueología, Edición provisional. Larache , Alta Comisaría de España en Marruecos.

Quintero Atauri, P. (1941): Apuntes sobre arqueología mauritana de la zona española, Compendio de noticias referentes a los descubrimientos arqueológicos efectuados en el siglo actual. Tetuán, Patronato de Investigación y Alta Cultura, Instituto General Franco.

Quintero Atauri, P.(1942): "Pinturas rupestres de Magara en Yebel Kasba". Archivo Español de Arqueología XV: 76.

Quintero Atauri, P. et Giménez Bernal, C. (1944): Excavaciones en Tamuda. Memoria resumen de las practicadas en 1943. Delegación de Educación y Cultura, $\mathrm{n}^{\circ} 7$. Tetuán, Alta Comisaría de España en Marruecos.

Orientaciones a los Interventores en la labor de Protectorado en Marruecos (1935). Tetuán, Delegación de Asuntos Indígenas.

Sánchez Pérez, A. (1926): La acción decisiva contra Abd el-Krim. Operaciones en el Rif central en colaboración con el ejército francés. Toledo, Sebastián Rodríguez Impresor.

Sánchez Pérez, A. (1948): "De arqueología". Mauritania 244: 63.

Sánchez Pérez, A.(1950-1951): “El Rif y los rifeños (1945), Aprovechamientos comunales y formas de cooperación en el Rif", dans Selección de Conferencias y Trabajos realizados durante el curso de Interventores: 95- 104.

Sánchez Pérez, A.(1951-1952): "Datos históricos sobre ciudades rifeñas", dans Selección de Conferencias y Trabajos realizados durante el curso de Interventores: $31-47$.
Sánchez Pérez, A. (1964): "Los moriscos de Hornachos, corsarios de Sale". Revista de Estudios Extremeños XX: 15.

Tarradell, M. (1949): "Estado actual de la investigación arqueológica en la zona de protectorado español en Marruecos", dans Crónica del IV Congreso Arqueológico del Sudeste Español (Elche, 1948): 80- 88. Publicaciones de la Junta Municipal de Arqueología y del Museo de Cartagena, Cartagena, IMprenta Española.

Touceda Fontenla, R. (1951): “Aixa Kandixa”. Mauritania: 92.

Touceda Fontenla, R. (1952): La fiesta de Moros y Cristianos de la Sainza en la Provincia de Orense. Tetuán, Imprenta del Majzén (= Archives Berbères (1953): Bulletin de l'Institut des Hautes Études Marocaines XL, 1-2 trimestres: 282-283).

Touceda Fontenla, R.(1954): "Oquedades en roca viva y paredes ciclópeas en Rfaif (Garbia)", dansI Congreso Arqueológico del Marruecos Español (Tetuán, 1953): 461-468. Tetuán, Imprenta Cremades.

Touceda Fontenla, R. (1954b): "Posible foco totémico en Marruecos", dans I Congreso Arqueológico del Marruecos Español (Tetuán, 1953): 511-514. Tetuán, Imprenta Cremades.

Touceda Fontenla, R. (1955): Los Hedaua de Beni Aros y su extraño rito. Tetuán, Editora Marroquí.

Touceda Fontenla, R. (1957a): "El paso de los árabes por la Rioja y la batalla de Clavijo". África XIV, 190: 421-423.

Touceda Fontenla, R. (1957b): "La Academia de Caballería. Notas para su historia". Revista de Historia Militar XIII, 26: 105-128.

Touceda Fontenla, R. (1958): "Marruecos, nuevo eslabón del hispanismo". Mauritania 363: 74-79.

Touceda Fontenla, R. (1959a): "Nuevos yacimientos arqueológicos romanos en la zona Norte de Marruecos". Mauritania 376: 99-107.

Touceda Fontenla, R. (1959b): "Dos islas españolas en la costa norte-africana". Mauritania 379: 222-224.

Touceda Fontenla, R. (1960): "Los ferrocarriles en Marruecos, I y II". Mauritania 397 et 398: 426-430 et $9-11$.

Touceda Fontenla, R. (1961): "Hagiografía musulmana. Sidi Muley Abdeslam ben Emchis; Sidi Heddi". Mauritania 403: 214-218; 404: 244-248; 405-406: 286-290.

Touceda Fontenla, R. (1962): “Conjeturas arqueológicas, las ruinas de Rfaif en la Cabila de Garbia. Il”. Mauritania 416-417: 241-245. 
Villanova, J. L. (2000): “La academia de interventores del Protectorado de España en Marruecos",dans II Coloquio Internacional de Estudios sobre África y Asia: 401-404. Melilla, Servicio de Publicaciones del Centro UNED- Melilla.

Villanova, J. L. (2002): "La formación de los interventores en el Protectorado español en Marruecos (1912- 1956)", dans El Protectorado español en Marruecos. Gestión colonial e identidades: $247-$ 280. Madrid, CSIC.

Villanova, J. L. (2005): "Los interventores del protectorado español en Marruecos (1912- 1956) como agentes geopolíticos". Eria 66: 93-111.

Villanova, J. L. (2006): Los interventores: la piedra angular del protectorado español en Marruecos. Barcelona, Bellaterra.

Villanova, J. L.(2008): “La cartografía elaborada por los interventores al finalizar la guerra del Rif: una herramienta al servicio del control político y militar en el Protectorado español en Marruecos".
Treballs de la Societat Catalana de Geografia 65: 507-519.

Villanova, J. L. (2010):“Cartographie et contrôle au Maroc sous le Protectorat espagnol (1912- 1956)”.M@ ppemonde 98: 2.

Villanova, J. L.(2012a): "Los interventores del protectorado español en Marruecos. Los principales agentes del desarrollo de la política colonial". Revista de Historia Militar, LVI, Número Extraordinario II: 161-201.

Villanova, J. L. (2012b): "Los interventores: el elemento clave del Protectorado español en Marruecos",dans Al servicio del Protectorado, España en Marruecos 1912- 1956. Madrid, Instituto de Historia y Cultura Militar, Ministerio de Defensa.

Villanova, J. L.(2013): “Obtener información: una de las principales funciones de los interventores del Protectorado español en Marruecos", dans La problemática colonial española en Marruecos: 69-100. Rabat, Instituto de Estudios Hispano-Lusos - Université Mohammed V. 